\title{
Delay in diagnosis of childhood asthma and its influence on respiratory consultation rates
}

\author{
Ian Charlton, Kevin Jones, John Bain
}

\begin{abstract}
A survey of cases of asthma in two general practices with a special interest in the disease identified 212 asthmatic children aged 0-16 years by the end of January 1989 who had been registered in that practice since birth. Delay in diagnosis, both in terms of time (median 2.95 years) and number of consultations (median 7), was considerable. Annual rates of consultations for respiratory symptoms that were initiated by the patients fell significantly after diagnosis (median before $=$ 1.80 , after $=0.95$ consultations/year), but the number initiated by the doctors rose significantly (median before $=0$, after $=1.01$ consultations/year). The overall rates before and after diagnosis were not significantly different (median before $=2.04$, after $=2.21$ consultations/year). Increased efforts are required to reduce this delay thereby minimising the morbidity of asthma in childhood.
\end{abstract}

The underdiagnosis of asthma in children was first highlighted by Speight in $1978 .^{1}$ Since then Levy and Bell have shown that even in their practice, which has a special interest in asthma, the median category of number of consultations for respiratory symptoms before a diagnosis of 'asthma' was made was $16-20^{2}$; the mean time between first respiratory consultation and diagnosis was 50.4 months (M Levy, personal communication). Jones and Sykes found a diagnostic delay of roughly $40 \%$ of the child's age. ${ }^{3}$

In this study we have examined delay in diagnosis, both in terms of time and numbers of consultations, and rates of consultation for respiratory symptoms before and after diagnosis.

\section{Subjects and methods}

Two practices with a special interest in asthma were studied: the urban practice of 8100 patients attached to the University Primary Medical Care Group in Southampton, and a training practice of 7990 patients in Aylsham, Norfolk. Only those children aged under 16 years who had been registered since birth were included. Disease registers were used to identify the asthmatic children in this group. For each eligible asthmatic child the date of birth, date of first respiratory consultation, date of diagnosis of asthma, and the number of consultations for respiratory symptoms before and after diagnosis (up to the end of January 1989), were recorded.

These consultations were divided into those initiated by the patient and those initiated by the doctor. Those initiated by the patient were defined as those consultations at which the patient had been recorded as presenting with one or more of the following: cough, wheeze, upper or lower respiratory tract infection, or sleep disturbed by coughing. Respiratory consultations initiated by the doctor were defined as those in which review was mentioned in the notes, those at a prescribed time after a previous visit (for example, 'see in two weeks'), or those where respiratory symptoms were reviewed when the patient attended for some other reason.

Delay in diagnosis was calculated by subtracting the age of first consultation for respiratory symptoms from that at which the word 'asthma' first appeared in the notes. The number of such consultations before diagnosis included both the first consultation and that at which the diagnosis was made. Annual rates for both types of consultation were calculated before diagnosis for patients whose delay in diagnosis was at least one year, and after diagnosis for those whose diagnosis had been made at least 12 months previously. This period was chosen for two reasons: firstly, to avoid the more recent period in which asthma clinics run by nurses were operating in both practices, and secondly, to avoid calculating annual rates based on less than one year's data. Rates before diagnosis were calculated by dividing the number of consultations up to and including that at which the diagnosis of asthma was made by the delay in diagnosis. Rates after diagnosis were calculated by dividing the number of respiratory consultations since diagnosis by the period between diagnosis and the end of the study. Data were analysed using the Statistical Package for the Social Sciences (SPSS) PC package for IBM compatible personal computers ${ }^{4}$ and the Confidence Interval Package of Gardner et al. ${ }^{5}$ The annual rates before and after diagnosis were compared by the Wilcoxon matched pairs signed rank test.

\section{Results}

A total of 212 children (123 boys and 89 girls) fulfilled the criteria for entry, 112 from Aldermoor and 100 from Aylsham. This gives a lifetime prevalence of asthma of $11 \%$ for Aldermoor and $10 \%$ for Aylsham. Table 1 shows the number of diagnoses of asthma made in 168 children diagnosed before the age of 8 years according to the year of diagnosis. This shows the time trend in the diagnosis of asthma for these younger children during the study period.

The median delay in diagnosis for the whole
Correspondence to: Dr Charlton.

Accepted 15 January 199 
Table 1 Time trend in diagnosis of asthma in 168 children aged $\leqslant 8$ years

\begin{tabular}{lcc}
\hline $\begin{array}{l}\text { Year of } \\
\text { diagnosis }\end{array}$ & \multicolumn{2}{c}{ Age at diagnosis (years) } \\
\cline { 2 - 3 } & $0-4$ & $4-8$ \\
\hline $1973-6$ & 2 & 0 \\
$1977-80$ & 5 & 7 \\
$1981-4$ & 20 & 25 \\
$1985-8$ & 55 & 54 \\
\hline Total & 82 & 86 \\
\hline
\end{tabular}

Table 2 Annual respiratory consultation rates before and after the diagnosis of asthma

$\begin{array}{ll}\text { Annual consultation rate } \\ \text { Mean (SD) } & \begin{array}{l}\text { Median } \\ \text { (interquartile range) }\end{array}\end{array}$

Before diagnosis $(n=158$, delay in diagnosis at least one year): Consultations initiated by

patient

Consultations initiated by

doctor

$2.29(1.72) \quad 1.80(1.04-3.03)$

Total consultations

$0.31(0.66) \quad 0(0-0.34)$

$2.60(2.08) \quad 2.04(1 \cdot 10-3 \cdot 12)$

After diagnosis $(n=156$, time since diagnosis at least one year): Consultations initiated by

patient

$1.38(1.36) \quad 0.95(0.41-1.86)$

Consultations initiated by

doctor

$1.46(1.42) \quad 1.01(0.43-2.03)$

Total consultations

$2 \cdot 84(2 \cdot 36) \quad 2 \cdot 21(1 \cdot 11-4 \cdot 12)$

group of 212 children was 2.95 years (95\% confidence interval (CI) 2.58 to 3.69 ) with the median number of consultations before diagnosis being 7 (95\% CI 6 to 8$)$. The median age at first presentation with respiratory symptoms was 0.8 years $(95 \%$ CI 0.69 to 1.02$)$. The rates of consultations initiated by the doctor and by the patient, and the total consultation rates for respiratory complaints before and after the diagnosis of asthma for the 158 children whose diagnosis had been delayed at least one year, and for the 156 children who had been followed up for at least one year since diagnosis are shown in table 2 .

In the 120 cases in which there had been a delay in diagnosis of more than a year, and a time since diagnosis of more than a year, the rate of consultations initiated by the patient after diagnosis was significantly less than it had been before diagnosis $(z=-6 \cdot 13, p<0.0001)$, but the rate initiated by the doctor after diagnosis was significantly greater than it has been before diagnosis $(z=-7.94, p<0.0001)$. There was no significant difference between the total rate before and after diagnosis $(\mathrm{z}=-0.56, \mathrm{p}=$ $0.58)$.

\section{Discussion}

Although our study - which was done in 1989cannot be compared directly with that of Levy and Bell (published in 1984), ${ }^{2}$ our results do indicate a trend towards a reduction in the total time period and the number of consultations for respiratory symptoms that occurred before the diagnostic label of 'asthma' is used in practices with an interest in the disease. The delay still remains substantial, however.

Does this delay matter? Firstly, our definition of delay must be somewhat arbitrary, because children may present with respiratory symptoms before they truly have 'asthma', which may exaggerate the period of real delay. Howie argued that general practitioners tend to proceed from symptoms to treatment without necessarily making a diagnosis. ${ }^{6}$ This is because general practitioners are often dealing with the early symptoms and signs of undifferentiated problems and precise diagnostic labels are often not justified. The importance of the label 'asthma' in ensuring correct treatment has, however, been clearly shown. ${ }^{7}$

In 1978 Speight examined 34 unselected patients who were referred to paediatric outpatient clinics in Newcastle and London and were diagnosed as having asthma. ${ }^{1}$ In only two children had asthma been diagnosed before referral, but most showed appreciable clinical improvement after diagnosis. He highlighted three factors that seemed to militate against the diagnosis of asthma being made in general practice. These were: parents' choice of symptoms, overemphasis on the role of infection, and reluctance to use the word 'asthma'.

In 1983 Speight et al conducted a large survey of 7 year old children at school in North Tyneside. ${ }^{8}$ Of 2700 children, 279 were reported by parents to have had a history of wheeze since they went to school. A sample of about two thirds $(n=179)$ was studied further. Only 21 had been diagnosed as having asthma, six after referral to hospital. Only a third of those who had more than 12 episodes of wheezing a year had been diagnosed as having asthma. By the end of the survey 31 children who were receiving prophylaxis had considerably reduced the amount of time that they spent off school.

A similar survey of 8 year olds in London was done in 1981 by Anderson et al; this also showed that wheezy children were being undertreated. ${ }^{7}$ Those said to have asthma were significantly more likely to have used antiasthmatic drugs than those who were not (56/94 compared with $8 / 185)$.

Our data have shown an almost exponential rise in the number of cases of asthma diagnosed, which although not related to total numbers of children in the practices, represents a substantial change in the diagnostic behaviour of the general practitioners concerned. From the available evidence, this increase should have resulted in a greater use of appropriate treatment and a possible reduction in morbidity. Further reduction in the substantial delays in diagnosis may increase this effect. There is likely, however, to be a period of delay during which the potential benefits of early treatment of asthma are outweighed by the risks of engendering anxiety and overtreatment. The magnitude of this 'optimal delay' is unknown.

Our results did not show that diagnosing asthma in a child reduces the total number of consultations for respiratory symptoms, but the desired endpoint is reduced morbidity, not reduced workload. It can be inferred, albeit indirectly, that some reduction in morbidity did occur after diagnosis, as rates of consultations initiated by the doctor (proactive) rose significantly and those initiated by the patient (reactive) fell significantly. This implies that some reactive or acute presentations may have been prevented by proactive care. To be certain that 
reducing diagnostic delay in childhood asthma in general practices leads to lowered morbidity requires a lengthy study relating delay to morbidity over time in that setting.

Clearly, merely labelling a child as 'asthmatic' cannot reduce morbidity, but if the application of the label leads to improved care in terms of greater attention to inhaler technique, proactive follow up by the nurse or doctor, the development of self management skills, and better explanation to parents, morbidity should be reduced. The nature of general practice may permit proper management of illnesses without precise diagnostic labelling, but we feel confident in recommending that in childhood asthma increased efforts should be made in primary care to further reduce diagnostic delay.

We thank Mark Mullee, senior statistical programmer, department of medical statistics and computing, University of South ampton, for help with the analysis, and the following members of the primary medical care group, Southampton University: Samantha Smith for help in data processing and Dr Roger Jones and $\mathrm{Dr}$ Ann-Louise Kinmonth for their encouragement and advice.

1 Speight ANP. Is childhood asthma being underdiagnosed and undertreated? BMF 1978;ii:331-2.

2 Levy M, Bell L. General practice audit of asthma in childhood. BMF 1984;289:1115-6.

3 Jones A, Sykes A. The effect of symptom presentation on delay in asthma diagnosis in children in a general practice. Respiratory Medicine 1990;84:139-42.

4 Norusis MJ. The Statistical Package for the Social Sciences (SPSS) guide to data analysis for SPSS/PC + . Chicago: SPSS Inc, 1989 .

5 Gardner MJ, Gardner SB, Winter PD. Confidence interval analysis (CIA), microcomputer program manual. London: British Medical Association, 1989.

6 Howie JGR. Diagnosis-the Achilles heel? $\mathcal{f} R$ Coll Gen Pract 1972;22:310-5.

7 Anderson HR, Bailey PA, Cooper JS, Palmer JC. Influence of morbidity, illness label and social, family and health service factors on drug treatment of childhood asthma. Lancet 1981;ii:1030-2.

8 Speight ANP, Lee DA, Hey EN. Underdiagnosis and undertreatment of asthma in childhood. $B M \mathcal{F} 1983 ; 286: 1253-5$.

Bird attack and campylobacter

Campylobacter jejuni is the commonest enteric bacterial pathogen in England and Wales. Most cases are sporadic and the source of infection is usually unknown but an outbreak in South Wales in May 1990 may have been caused by the contamination of milk by birds (Southern et al, Lancet 1990;336:1425-7).

In a case-control study involving 32 cases and 64 controls significantly more cases reported that their milk bottles had been attacked by birds especially in the week before the onset of illness. Controls were less likely to have their milk delivered to the door and more likely to have discarded milk from attacked bottles or to have taken measures to protect the bottles from birds.

The suspected birds are magpies and jackdaws. Campylobacter spp have been grown from the beaks and cloacae of these birds. You can hazard a guess at what kind of bird has been at your milk by looking at the bottle top. Tits, it seems, peck holes in the shiny bottle top but magpies tear off the top completely.

How many paediatricians know that the word pica is taken from the latin for magpie (Pica pica)? Alfred Hitchcock devotees, of course, have known for a long time that the birds are out to get us. 